\title{
Diagnostic utility of Immunohistochemical expression of HE4 in Epithelial Ovarian Neoplasm
}

\author{
Begum Afrin Nahar ${ }^{1}$, Rama Saha ${ }^{2}$, Chhanda Das ${ }^{2 *}$ and GourishankarKamilya ${ }^{3}$ \\ ${ }^{1}$ Dept of Pathology, IPGME\&R, Kolkata, India \\ ${ }^{2}$ Dept of Pathology, IPGME\&R, Kolkata \\ ${ }^{3}$ Department of Obstetrics and Gynaecology,IPGME\&R, Kolkata
}

\begin{abstract}
Background: Mortality in case of ovarian malignancy is high due to late diagnosis. Early and accurate diagnosis can improve the case specific management. HE4 (human epididymis protein 4) which is proved to be overexpressed in the ovarian cancer cells, is considered a new biomarker for ovarian cancer diagnosis.
\end{abstract}

Aims and objectives: The study was undertaken toevaluate the immunohistochemical expression of HE4 in various epithelial ovarian neoplasm.

Material and Methods: It was a cross sectional, prospective,single institution based study,conducted in the department of Pathology in collaboration with the Department of Gynecology and Obstetrics, from December 2016 to January 2019 in our institution. A total 71 cases were selected for this study.

Results: Serous tumour was the most common epithelial tumour followed by mucinous tumour. Highest percentage of expression of HE4 was seen in high grade serous cancer and malignant endometrioid tumour. Benign serous and mucinous tumour shows negative expression mostly. We foundStatistically significant correlation between HE4 expression and different types of epithelial ovarian tumours ( $\mathrm{p}=0.000)$.

Conclusions: HE4 was highly expressed in malignant ovarian tumour especially serous and endometrioid carcinomaand can be used as an important biomarker for malignant ovarian neoplasm. Expression in high grade ovarian serous cancer support its prognostic value also.

\section{Keywords: Biomarker, Epithelial Ovarian Neoplasm, HE4}

\section{Introduction}

Ovarian carcinoma has the highest mortality rate among all gynaecological malignancies. It is the sixth most common cancer in women worldwide.[1] WHO classified ovarian tumours into 5 types, of which surface epithelial tumour is the most common. WHO recognizes seven histological subtype of epithelial ovarian tumour.[2] Serous carcinoma which is of epithelial origin, the most common type of ovarian carcinoma carries the poorest prognosis, make upto $30-70 \%$ of all diagnosis.[3] Ovarian carcinoma most often diagnosed late due to their subtle and undetected clinical symptoms,[4] and this leads to high mortality rate.[5] Though ultrasound is a routinely performed imaging technique, high levels of expertise is required to differentiate between benign and malignant ovarian tissues. Hence there is a need to develop biomarkers for early diagnosis.

HE4 Human Epididymis protein 4, also known as WAP type four disulfide core (WFDC2), is one of the most promising bio markers for early detection. HE4 is primarily expressed in the reproductive and respiratory tracts [6] and is over expressed in ovarian cancer cells, especially in histologic subtypes of serous and endometrioid carcinoma and it has been suggested to be a serological marker also. [7] The serologic detection of HE4 has most recently been shown to have increased sensitivity and specificity for the detection of ovarian cancer, compared with targeting CA125, the current gold standard serum bio-marker for screening metastatic ovarian carcinomas. [8] Several studies revealed a positive correlation between serum level and IHC expression of HE4.In this study we aim to evaluate the histolopathogical spectrum of epithelial ovarian neoplasm, and to study the immunohistochemical expression of HE4 in different histopathological types and subtypes of ovarian neoplasm.

\section{Material and Methods}

Study was conducted with oophorectomy specimen with or without uterus, fallopian tube and omentum obtained from the Gynaecology and Obstetrics department in our Institution after obtaining approval from Ethics Committee from December 2016 to June 2018. All specimens received for histopathological sampling was fixed in $10 \%$ neutral buffered formalin solution and embedded in paraffin. The formalin fixed, paraffin embedded tissue blocks were sliced 
into 4-5 microns sections which were subsequently stained with hematoxylin and eosin.HE4 antibody is a rabbit monoclonal antibody derived from cell culture supernatant that is concentrated, dialyzed, filter sterilized and diluted in buffer $\mathrm{pH} 7.5$, containing BSA and sodium azide as a preservative. Immunohistochemical staining with HE4 antibody done following antigen retreival in microwave method.Human epididymis was taken as positive (with antibody) and negative (without antibody) control.

Interpretation of immunostain Scoring system :Cytoplasmic staining was graded for intensity (0negative,1- weak positive, 2-moderate, and 3- strong) and percentage of positive cell [0, 1(1-24\%), 2 (25-49\%), and $3(50-100 \%)$. The grades were multiplied to determine an $\mathrm{H}$ score. Protein expression was then defined as negative $(\mathrm{H}$ score $=0$ ), weak ( H score $=1-3$ ), or strong ( H score $>4)$.[9]

Statistical analysis: Data has been analysed by appropriate statistical tests using statistical software SPSS 20.0 (IBM Armonk, New York, USA). Microsoft Excel worksheet has been used to record the relevant demographic, clinical, laboratory data. Records has been kept confidential and available only to the authorized staff. Two tailed P-value $<0.05$ has been considered indicative of statistical significance.

\section{Results}

71 epithelial ovarian tumour were analyzed in our study. Age of patients range from 14-80 yrs. Most common age group 41-60 yrs. 53\% patients presents with lower abdominal pain. $42(59 \%)$ cases found benign and 27 $(38 \%)$ cases are malignant. $2(3 \%)$ cases diagnosed as borderline tumour. Patients with benign disease are mostly premenopausal (71\%) whereas malignant cases are mostly postmenopausal $(63 \%)$. Serous tumour is the most common (60\%) epithelial ovarian tumour followed by mucinous tumour $(27 \%)$. Of the epithelial ovarian carcinoma serous carcinoma is the most common (67\%), followed by endometrioid (16\%) variety. (Table 1$)$

HE4 was highly expressed in serous ovarian cancer, high grade shows $100 \%$ (13/13) strong positivity, whereas low grade serous cancer shows $60 \%(3 / 5)$ strong positivity and $20 \%(1 / 5)$ are weakly positive, endometrioid adenocarcinoma shows 80\% (4/5) strong positivity. Lower level of expression is seen in mucinous cancer, $50 \%$ weakly positive and $50 \%$ negative, clear cell carcinoma one case found which shows weak positivity. (Fig1,2,3,4) Benign tumour shows weak expression (Table 2). Statistically significant difference of HE4 expression pattern seen in different types of epithelial ovarian tumour $\mathrm{P}$ value $<.05$. (Table 3)

Statistically significant different pattern of expression of HE4 is seen in different types of epithelial ovarian tumour $(\mathrm{P}$ value $<.05)$.

\section{Discussion}

Search is ongoing for many years to have a novel more sensitive and more specific tumour marker or diagnostic algorithm to classify patient with a pelvic mass and to

Table 1: Epithelial ovarian tumour in to different types $(n=71)$.

\begin{tabular}{|l|c|}
\hline Tumour type & No \\
\hline Serous tumour - a) benign & a) 24 \\
b) borderline & b) 1 \\
c) malignant & c) 18 \\
\hline Mucinous tumour - a) benign & a) 16 \\
b) borderline & b) 1 \\
c) malignant & c) 2 \\
\hline Endometriod tumour- malignant & 5 \\
\hline Clear cell tumour- malignant & 1 \\
\hline Brenner tumour with mucinous tumour & 1 \\
\hline Seromucinous tumour & 1 \\
\hline Mixed epithelial and mesenchymal tumour & 1 \\
\hline Total & 71 \\
\hline
\end{tabular}

Table 2: HE4 score of 71 epithelial ovarian tumour.

\begin{tabular}{|l|l|l|l|l|}
\hline Histological type & Total no & $\begin{array}{l}\text { Weak positive } \\
\text { ( H score=1-3) }\end{array}$ & $\begin{array}{l}\text { Strong positive } \\
\text { ( H score } \mathbf{4})\end{array}$ & $\begin{array}{l}\text { Negative } \\
\text { (H score = 0) }\end{array}$ \\
\hline Benign serous tumour & 24 & $2(8 \%)$ & 0 & $22(92 \%)$ \\
\hline Borderline serous tumour & 1 & 0 & 0 & $1(100 \%)$ \\
\hline $\begin{array}{l}\text { Malignant serous tumour a) low grade } \\
\text { b) high grade }\end{array}$ & 05 & $\begin{array}{l}1(20 \%) \\
0\end{array}$ & $\begin{array}{l}03(60 \%) \\
13(100 \%)\end{array}$ & $\begin{array}{l}01(20 \%) \\
0\end{array}$ \\
\hline
\end{tabular}




\begin{tabular}{|l|l|l|l|l|}
\hline Histological type & Total no & $\begin{array}{l}\text { Weak positive } \\
\text { ( H score=1-3) }\end{array}$ & $\begin{array}{l}\text { Strong positive } \\
\text { ( H score } 4)\end{array}$ & $\begin{array}{l}\text { Negative } \\
\text { (H score = })\end{array}$ \\
\hline Benign mucinous tumour & 16 & $1(6 \%)$ & 0 & $15(94 \%)$ \\
\hline Borderline mucinous tumour & 1 & 0 & 0 & $01(100 \%)$ \\
\hline Malignant mucinous tumour & 2 & $1(50 \%)$ & 0 & $1(50 \%)$ \\
\hline Endometriod tumour- malignant & 5 & 0 & $4(80 \%)$ & $1(20 \%)$ \\
\hline Clear cell tumour- malignant & 1 & $1(100 \%)$ & 0 & 0 \\
\hline Benign brenner with mucinous tumour & 1 & 0 & 0 & $1(100 \%)$ \\
\hline Benign seromucinous tumour & 1 & 0 & 0 & $1(100 \%)$ \\
\hline Mixed epithelial and mesenchymal tumour & 1 & $1(100 \%)$ & 0 & 0 \\
\hline Total & $\mathbf{7 1}$ & $\mathbf{8}$ & $\mathbf{2 0}$ & $\mathbf{4 3}$ \\
\hline
\end{tabular}

Table 3: Chi square test (using IBM, SPSS statistic version 20)

\begin{tabular}{|l|c|c|c|}
\hline & Value & df & Asymp. Sig.(2sided) \\
\hline Pearson Chi square & 84.950 & 22 & .000 \\
Likelihood ratio & 90.208 & 22 & .000 \\
N of Valid Cases & 71 & & \\
\hline
\end{tabular}

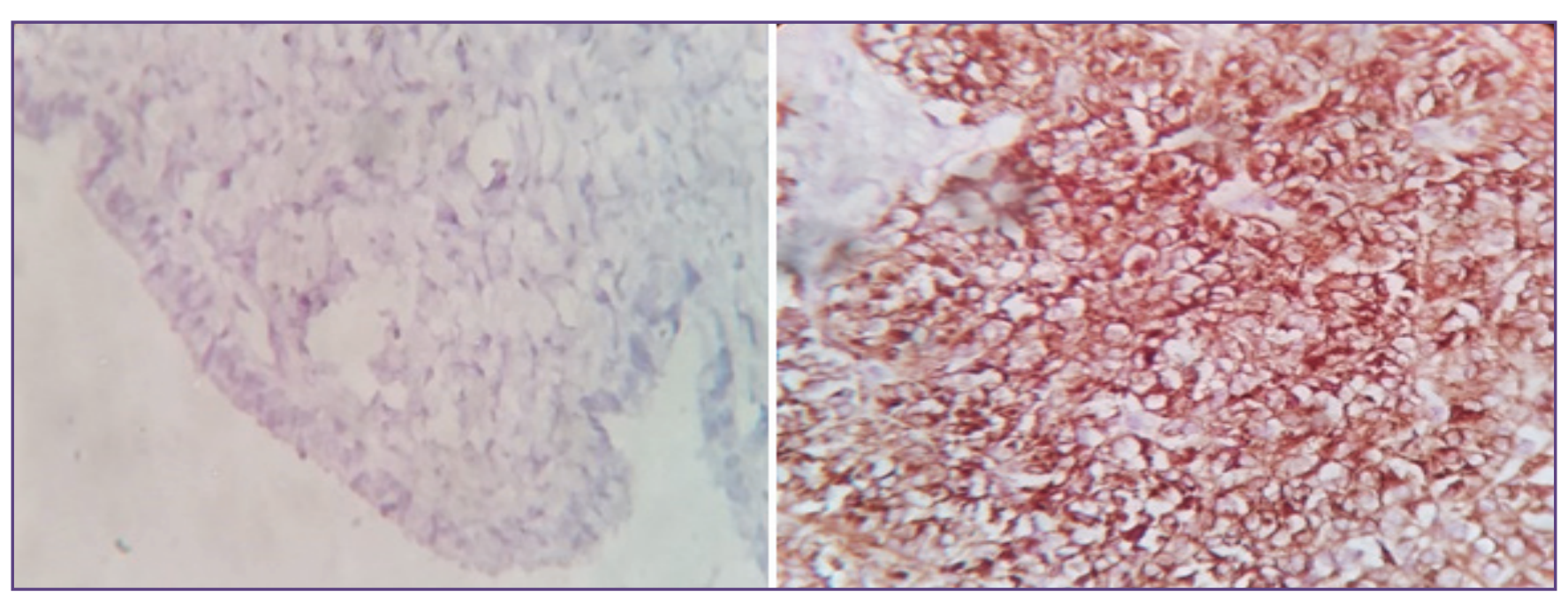

Fig. 1: Negative staining, serous cystadenoma (400X)Fig 2.Strong cytoplasmic positivity in High gradeserous carcinoma( 400X)

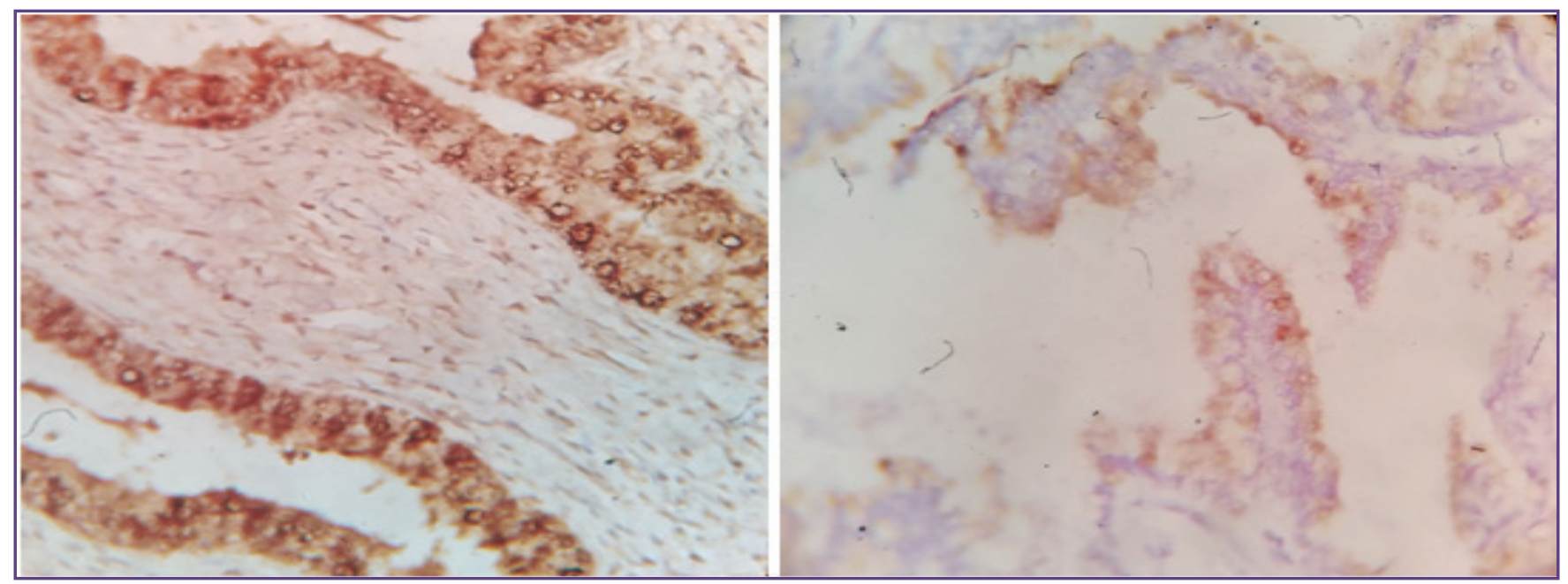

Fig. 3: Strong cytoplasmic positivity in Low gradeFig 3Weak cytoplasmic positivity in Mucinous serous carcinoma( 400X)carcinoma, 
screen ovarian cancer. In1999 the human epididymis secretory protein 4 (HE4) gene was found to be over expressed in ovarian cancer. [10]The first report mentioning HE4 as a potential serum biomarker for ovarian cancer was published in 2003. [11]

ROMA (Risk of Malignancy Algorithm) based on CA125 and the novel HE4 marker has recently emerged as a promising approach to the preoperative categorization of malignancy risk. The diagnostic performance of ROMA was advocated for the first time by Moore et al. Moore et al $(2008,2009)$ published a series of papers that use a combination of CA125, HE4 and menopausal status to predict the presence of a malignant ovarian tumour (ROMA). [12]Recently, a study showed HE4 is more specific than CA125 in benign \& malignant condition. [13] HE4 serum level may be abnormal mainly in patient with renal failure or effusion in patient with lung cancer or with chronic liver disease. HE4 protein is frequently over expressed in ovarian cancer, especially in serous and endometrioid histology. [7] However HE4 is not a specific of ovarian cancer \& some expression has also been found in other malignancy mainly pulmonary and endometrial carcinoma. [13]

In our study, age of the patient ranges from 14-80 years. This is similar to the studies of Agarwal P et al where the age range of patients was from 12-80 years.[14] Most of the patient of our study presented with lower abdominal pain (53\%). Lurie $\mathrm{G}$ et al, observed abdominal pain as the most common symptoms among ovarian cancer patients. [15] In our study $59 \%$ cases found benign and $38 \%$ cases are malignant $3 \%$ cases diagnosed as borderline tumour. Patients with benign disease are mostly premenopausal (71\%) whereas malignant cases are mostly postmenopausal (63\%). Maheshwari et al also reported $71.9 \%$ benign neoplasms constituting the largest group along with $23.7 \%$ malignant neoplasms and 4.4\% borderline neoplasms.[16] Studies by Siddiqui R B et al also had similar findings. [17] In our study the most common type of epithelial tumor was serous tumor $(60 \%)$ followed by mucinous tumour (27\%)]. In our study of the epithelial ovarian carcinoma serous carcinoma is the most common (67\%), followed by endometrioid (16\%) variety. This finding was however dissimilar with Maheshwari et al who reported that mucinous cyst adenocarcinomas constituted the largest group among malignant neoplasm.[16]

100 ovarian tumour stained with HE4 antibody of which $89 \%(54 / 61)$ benign tumour shows negative expression, $100 \%(2 / 2)$ borderline tumour shows negative expression. 95\% (35/37) malignant tumour shows positive expression in our study (Table 2).
Drapkin et al study found that expression of HE4 was closely related with tissue type of ovarian cancer.[7] In our study high grade serous cancer showed higher expression than low grade serous cancer $(60 \%$ strong positivity in low grade in comparison with $100 \%$ positivity in high grade). Another study conducted by Li-e Zheng et al found high expression $(100 \%)$ in serous ovarian cancer, $66 \%$ in mucinous ovarian cancer, $66 \%$ in clear cell cancer, $100 \%$ in endometrioid cancer. [18] Our study showed that HE4 is highly expressed in serous ovarian cancer ( $100 \%$ in high grade) and endometroid cancer (80\%). Low level of expression is seen in mucinous cancer, clear cell cancer in our study. Benign tumour shows very low level of expression (mucinous 6\% and serous 8\%). Study by F Rahmat and Hairuszah Ithnin (2017) on Immunohistochemical Expression of HE4 in Ovarian Serous Carcinoma, found $100 \%$ positive staining in women $<40$ yrs old,among women above 40 yrs $98 \%$ show positive staining and only one negative staining to HE4 in ovarian serous carcinoma, which support our study. [19] Study by Penelope Georgakopoulos et al showed HE4 protein expression was $42 \%$ in serous cystadenomas, $100 \%$ in serous borderline tumour and $100 \%$ in serous cancer, intensity of HE4 staining was strong in majority of serous carcinoma, but was either weak or negative in serous cystadenoma. [20] Expression of high grade serous carcinoma correlate with our study which also show $100 \%$ positive expression, but it does not correlate with expression of benign or borderline serous tumour.HE4 Expression of serous carcinoma of our study also correlate with study of T Bulut et al who found 90.32\% HE4 tissue expression among ovarian serous carcinoma, and found it is superior to CA 125.[21]

\section{Conclusions}

Serous tumour is the most common epithelial ovarian tumour and serous carcinoma is the most common epithelial ovarian carcinoma. HE4 is highly expressed in certain types of ovarian cancer and can be used as biomarker for ovarian cancer particularly of serous (the most common ovarian canecr) and endometiod variety. Low level of expression is seen in mucinous cancer, clear cell cancer in our study, but because of the limited cases of these subtype of tumour, the conclusion may be different in general and needs further study.

\section{Limitations}

Serum HE4 level estimation before surgery would help us to correlate with the tissue expression of HE4 which is lacking in this study. Little is known about the specific biological processes that are involved in the regulation of HE4 expression. Literature search reveals no study in this field in our region. According to study design and short duration of study period, follow up of cases was not possible. 


\section{References}

1. Smith TJ, Temin S, Alesi ER, Abernethy AP, Balboni TA, Basch EM et al. American Society of Clinical Oncology Provisional Clinical Opinion: The Integration of Palliative Care Into Standard Oncology Care". Journal of Clinical Oncology 30 (8): 880-887.

2. WHO Classification of Tumours of Female Reproductive Organs, In: Robert J Kurman, Maria Lusia Carcangiu, C.Simon Herrington, Robert H. Young ed.4th,France, IARC, Lyon: IARC,2014 p 12-13.

3. Nolen B, Marrangoni A, Velikokhatnaya L, Prosser D, Winans M, Gorelik E, Anna LA: A serum based analysis of ovarian epithelial tumorigenesis. Gynecol Oncol 2009, 112(1): 47-54.

4. Pettersson F. Annual Report on the Results of Treatment in Gynecological Cancer, FIGO. Int J Gynaecol Obstet 1994;22:83-102.

5. Ozols RF. Update on the management of ovarian cancer. Cancer J 2002;8:( 1): 22-30.

6. Galgano MT, Hampton GM, Frierson HF, Jr. Comprehensive analysis of HE4 expression in normal and malignant human tissues. Mod Pathol 2006;19:847-53.

7. Drapkin R, von Horsten HH, Lin Y, et al. Human epididymis protein 4 (HE4) is a secreted glycoprotein that is overexpresse by serous and endometrioid ovarian carcinomas. Cancer Res 2005;65:2162-9.

8. Anastasi E, Marchei GG, Viggiani V, et al. HE4: a new potential early biomarker for the recurrence of ovarian cancer. Tumour Biol 2010;31:113-9.

9. Bulut.T, Celik. B, A.D. Yalcin, S. Keser. Tissue expression of HE4 and its correlation with CA125 and p53 in high grade serous ovarian carcinoma. European journal of Gynaecological Oncology. January 2018.

10. Schummer M, NgWV, Bumgarner RE,Nelson PS, Schummer B, BednarskiDW et al Comparative hybridization of an array of 21500 ovarian cDNAs for the discovery of genes overexpressed in ovarian carcinomas. Gene 1999; 238: 375-385.

11. Hellstrom I, Raycraft J, Hayden-Ledbetter M, Ledbetter JA, Schummer M, McIntosh M et al. The HE4 (WFDC2) protein is a biomarker for ovarian carcinoma. Cancer Res 2003; 63: 3695-3700.
12. Moore RG, McMeekin DS, Brown AK, DiSilvestro P, Miller $\mathrm{MC}$, Allard WJ et al. A novel multiple marker bioassay utilizing HE4 and CA125 for the prediction of ovarian cancer in patients with pelvic mass.Gynec Oncol.2009;(112):40-6.

13. Escudero JM, Auge JM, Filella X, Torne A, Pahisa J, Molina R.The utility of serum human epididymis protein 4 (HE4) in patients with malignant and non malignant diseases: comparison with CA125. Clin Chem. 2011 (in press)

14. Agarwal P, Kulkarni DG, Chakrabarti PR, Chaurasia S, Dixit M, Gupta K. Clinicopathological Spectrum of Ovarian Tumors: A 5-Year Experience in a Tertiary Health Care Center. Journal of Basic and Clinical Reproductive Sciences, 2015;4(2).

15. Malik I.A. A Prospective Study of Clinico-pathological Features of Epithelial Ovarian Cancer in Pakistan. JPMA 2002;52:155.

16. Maheshwari V, Tyagi SP, Saxena K, Tyagi N, Sharma R, Aziz M, Hameed F. Surface epithelial tumours of the ovary. Indian J Pathol Microbiol. 1994;37(1):75-85.

17. Siddiqui RB, Shankar B, Khan IM. Morphological Patterns of Ovarian Neoplasms in Different Age Groups - A Center based study. P J M H S 2015;9(4): 1139.

18. Li-e Zheng, Jun - ying Qu, Fei He. The diagnosis and pathological value of combined detection of HE4 and CA125 for patients with ovarian cancer. Open Med 2016; 11: $125-132$

19. Rahmat F, Ithnin H Immunohistochemical Expression of Human Epididymis 4 (He4) in Ovarian Serous Carcinoma in Hospital Serdang from the Year 2006- 2013. Gynecol Obstet (Sunnyvale) 2017; 7: 453. doi: 10.4172/21610932.10004531

20. Penelope Georgakopoulos, Saira Mehmood, Ali Akalin Kenne tShroyer Immunohistochemical Localization of HE4 in Benign, Borderline, and Malignant Lesions of the Ovary. International Journal of Gynecological Pathology 31:517523.

21. Bulut.T, Celik.B, A.D.Yalcin, S. Keser. Tissue expression of HE4 and its correlation with CA125 and p53 in high grade serous ovarian carcinoma.European journal of GynaecologicalOncology. 2017 ISSN: 0392-2936 XXXVIII, n. 5, 2017 doi: 10.12892/ejgo3992.2017

*Corresponding author:

Dr Chhanda Das, 31 Eastern park, First Road, Santoshpur, Kolkata 75 INDIA

Phone: +919433116223

Email: chhhdas@gmail.com

Financial or other Competing Interests: None. 